\title{
The octagon as a determinant
}

\author{
Ivan Kostov, ${ }^{a}$ Valentina B. Petkova ${ }^{b}$ and Didina Serban ${ }^{a}$ \\ ${ }^{a}$ Institut de Physique Théorique, DSM, CEA, URA2306 CNRS, \\ Saclay, Gif-sur-Yvette F-91191, France \\ ${ }^{b}$ Institute for Nuclear Research and Nuclear Energy, Bulgarian Academy of Sciences, \\ 72 Tsarigradsko Chaussee, Sofia 1784, Bulgaria \\ E-mail: ivan.kostov@ipht.fr, petkova@inrne.bas.bg, \\ didina.serban@ipht.fr
}

ABSTRACT: The computation of a certain class of four-point functions of heavily charged BPS operators boils down to the computation of a special form factor - the octagon. In this paper, which is an extended version of the short note [1], we derive a non-perturbative formula for the square of the octagon as the determinant of a semi-infinite skew-symmetric matrix. We show that perturbatively in the weak coupling limit the octagon is given by a determinant constructed from the polylogarithms evaluating ladder Feynman graphs. We also give a simple operator representation of the octagon in terms of a vacuum expectation value of massless free bosons or fermions living in the rapidity plane.

KEYwords: AdS-CFT Correspondence, Integrable Field Theories

ARXIV EPRINT: 1905.11467 


\section{Contents}

1 Introduction $\quad 1$

1.1 The simplest 4-point correlation function 3

1.2 The octagon form factor 4

$\begin{array}{lll}1.3 & \text { Summary of the results } & 7\end{array}$

$\begin{array}{lll}\text { 1.3.1 The octagon } & \text { Tas a determinant } & 7\end{array}$

$\begin{array}{lll}1.3 .2 & \text { Perturbative expansion } & 8\end{array}$

$\begin{array}{ll}\text { 1.3.3 The null-square limit } & 9\end{array}$

2 The octagon as a pfaffian $\quad 9$

2.1 The octagon as a Fredholm pfaffian 9

\begin{tabular}{ll}
2.2 & Summing up the bound states \\
\hline
\end{tabular}

$\begin{array}{lll}2.3 & \text { From Fredholm kernel to a semi-infinite matrix } & 14\end{array}$

$\begin{array}{lll}2.4 & \text { Integral representation for the matrix elements } & 15\end{array}$

3 The perturbative octagon $\quad 16$

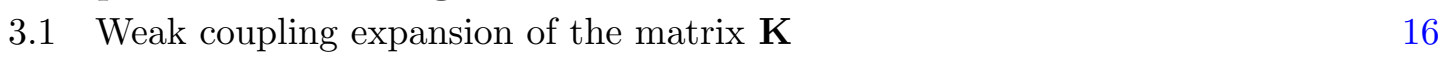

$\begin{array}{lll}3.2 & \text { The perturbative octagon as a determinant } & 17\end{array}$

$\begin{array}{lll}3.3 & \text { Expansion to nine loops } & 17\end{array}$

$\begin{array}{llr}3.4 & \text { Fishnets } & 18\end{array}$

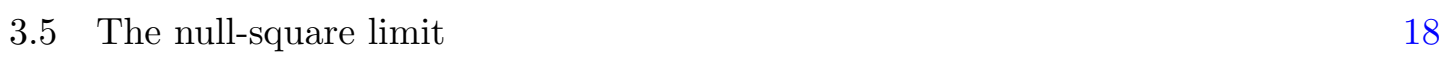

4 CFT representation $\quad 19$

4.1 The octagon in terms of a free chiral boson 20

$\begin{array}{lll}4.2 & \text { Real fermion } & 21\end{array}$

5 Conclusions $\quad 22$

$\begin{array}{ll}\text { A Perturbative evaluation of the matrix elements } & 23\end{array}$

\section{Introduction}

In this paper we address the computation of the octagon form factor which appears as a building block for the evaluation of a class of four-point correlation functions of singletrace half-BPS operators in $\mathcal{N}=4$ planar SYM [2]. We are doing so using integrabilityinspired techniques [3-6], and in particular the recently proposed geometric decomposition of correlation functions into hexagons [7-12]. The hexagons are form factors for non-local operators creating curvature defects. While the three-point correlation functions of halfBPS operators are trivial, the four-point function of such operators have a rather rich structure. 
As pointed out in [2], in certain cases these correlation functions can be evaluated exactly. In particular this is the case in the limit of half-BPS operators with large Rcharges and specially tuned polarisations the correlation function factorises into a sum of products of two octagon form factors, or octagons. From the point of view of the dual string theory, the octagon is an off-shell open string partition function with classical boundaries representing geodesics in $\mathrm{AdS}_{5}$. The closed string worldsheet representing a sphere with four punctures contains four consecutive classical geodesics. Such a worldsheet splits into two open-string worldsheets spanned on the four geodesics. Very recently it was discovered that similar factorisation occurs in any given order of the $1 / N_{c}$ expansion [13].

In this paper we obtain a closed analytic expression of the octagon at any coupling in terms of a Fredholm pfaffian. ${ }^{1}$ This allows us to represent the square of the octagon as a Fredholm determinant. To compute this Fredholm determinant, we write the Fredholm kernel $K(u, v)$ on a basis of functions $\psi_{n}(u)$,

$$
K(u, v)=\sum_{m, n=0}^{\infty} \psi_{m}(u) T_{m n} \psi_{n}(v) .
$$

with certain matrix operator $T$ and further factorise by Fourier transform. If we restrict the sum to a finite "cutoff" $N$, the Fredholm deterrminant becomes a usual $N \times N$ determinant. It can happen, and this is the case in our problem, that the Fredholm determinant is given by the limit $N \rightarrow \infty$ of the ordinary determinant. This procedure is similar to the method of degenerate kernels [15] used to solve integral equations. It is also possible to approximate the Fredholm pfaffian by ordinary pfaffians by applying the pfaffian integration theorem $[16,17]$. The advantage of this manipulation is that the multiple integrals break into sums of products of simple integrals.

We claim that to any loop order the pfaffian can be recast as a determinant of another matrix. From the determinant representations one confirms by direct computation the conjecture of [18] that the perturbative expansion of the octagon can be recast as a multilinear combination of ladder integrals

$$
\mathbb{O}_{\ell}=1+\sum_{n=1}^{\infty} \mathcal{X}_{n}(\phi, \varphi, \theta) \sum_{J=n(n+\ell)}^{\infty} \sum_{j_{1}+\ldots j_{n}=J} c_{j_{1}, \cdots j_{n}} f_{j_{1}} \cdots f_{j_{n}} g^{2 J} .
$$

In this sum the dependence on the polarisations is carried by the weight factors $\mathcal{X}_{n}[8$, 10], to be recalled in the following, the coefficients $c_{j_{1} \ldots j_{n}}$ are rational numbers, and the (conveniently normalised) ladder integrals are given, for $|z|<1$, by

$$
\begin{aligned}
f_{k}(z, \bar{z}) & \equiv k !(k-1) ! F_{k}(z, \bar{z}) \\
& =\sum_{j=k}^{2 k} \frac{(k-1) ! j !}{(j-k) !(2 k-j) !}(-\log z \bar{z})^{2 k-j} \frac{\operatorname{Li}_{j}(z)-\operatorname{Li}_{j}(\bar{z})}{z-\bar{z}} .
\end{aligned}
$$

The form (1.2) of the perturbative octagon carries some resemblance to the result of Basso and Dixon [19] obtained for the fishnet limit of the $\mathcal{N}=4$ SYM [20]. ${ }^{2}$ The analytic

\footnotetext{
${ }^{1}$ The fact that the sum over virtual particles can be written as a Fredholm pfaffian has been noticed before in [14].

${ }^{2}$ The integrability of the fishnet Feynman graphs has been first established by A. Zamolodchikov [21].
} 
expression obtained in [19] for the fishnet has the form of a single determinant of ladders, while bootstraping the Ansatz (1.2) one obtains [18] a series in the minors of the semiinfinite matrix

$$
\mathbf{f}=\left(\begin{array}{ccc}
f_{1} & f_{2} & f_{3} \\
f_{2} & f_{3} & f_{4} \\
f_{3} & f_{4} & f_{5} \\
\cdot & \cdot & \cdot
\end{array}\right)
$$

Our explicit solution for the perturbative octagon is in the form of either pfaffian or determinant, of semi-infinite matrices with elements represented by series of ladder integrals. This gives in particular an explicit analytic expression for the coefficients $c_{j_{1}, \cdots j_{n}}$ in the expansion (1.2). Finally, we give an alternative derivation of the pfaffian and the determinant representations based on an operator construction of the octagon with free real massless Majorana fermions living on the Zhukovsky plane. ${ }^{3}$

The paper is organised as follows. In the rest of this section we present the setup and the results. Section 2 gives formulation of the octagon as a Fredholm pfaffian. Section 3 is devoted to the perturbative analysis. Section 4 gives the CFT fermionic representation of the octagon.

\subsection{The simplest 4-point correlation function}

A half-BPS field is characterised by its position $x$ in the Minkowski space, a null vector $y$ giving its $\mathfrak{s o}(6)$ polarisation, and its scaling dimension $K$,

$$
\mathcal{O}_{i}=\operatorname{Tr}\left[\left(y_{i} \cdot \Phi\left(x_{i}\right)\right)^{K}\right] .
$$

The correlation function for four such operators can be computed non-perturbatively by the hexagonalisation method designed first in [7] for the computation of the three-point functions and adjusted for the four-point functions in [8-10]. The sum over virtual states prescribed by the hexagonalisation simplifies for heavy fields (large $K$ ) and particular choices of the polarisations because some of the channels of propagation of the virtual particles are suppressed. In the correlators analysed in [2] the sum over virtual particles factorises into a sum of products of two octagons. An octagon $\mathbb{O}_{\ell}(g, x, \bar{z}, \alpha, \bar{\alpha})$, sketched in figure 1, is composed of two hexagons glued together by inserting a complete set of virtual states. It depends only on the bridge length $\ell$ between the two hexagons, 't Hooft coupling $g$ and the cross ratios (1.6) in the coordinate and in the flavour spaces

$$
\begin{array}{rlrl}
z \bar{z} & =\frac{x_{12}^{2} x_{34}^{2}}{x_{13}^{2} x_{24}^{2}}=u, & (1-z)(1-\bar{z})=\frac{x_{14}^{2} x_{23}^{2}}{x_{13}^{2} x_{24}^{2}}=v \\
\alpha \bar{\alpha}=\frac{\left(y_{1} \cdot y_{2}\right)\left(y_{3} \cdot y_{4}\right)}{\left(y_{1} \cdot y_{3}\right)\left(y_{2} \cdot y_{4}\right)}, & (1-\alpha)(1-\bar{\alpha})=\frac{\left(y_{1} \cdot y_{4}\right)\left(y_{2} \cdot y_{3}\right)}{\left(y_{1} \cdot y_{3}\right)\left(y_{2} \cdot y_{4}\right)}
\end{array}
$$

\footnotetext{
${ }^{3}$ By Zhukovsky plane we mean the rapidity plane with two simple branch points at $u= \pm 2 g$. A similar operator representation has been proposed in [22] for the three-point function of non-BPS operators.
} 


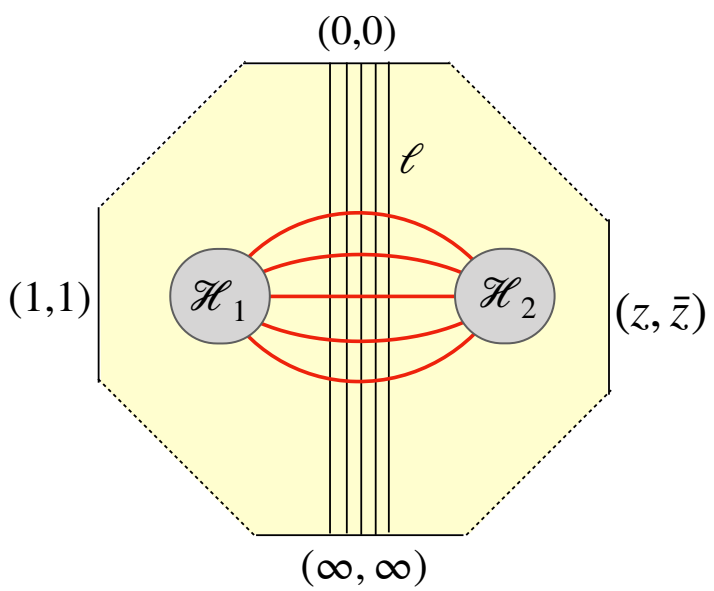

Figure 1. A sketch of the octagon $\mathbb{O}_{\ell}$. The parameters $z$ and $\bar{z}$ fix the conformal geometry of the octagon. The red lines symbolise the mirror particles propagating between the two hexagons, each one characterised by a rapidity $u$ and a bound state number $a$. Each mirror particle has to pass across a 'bridge' composed of $\ell$ Wick contractions.

The simplest four-point function that leads to such a factorisation, named in [2] the simplest, is characterised by $\left(y_{1} \cdot y_{4}\right)=\left(y_{2} \cdot y_{3}\right)=0$. For example (the dots stand for the sum over permutations)

$$
\begin{aligned}
\mathcal{O}_{1}(0) & =\operatorname{tr}\left(Z^{\frac{K}{2}} \bar{X}^{\frac{K}{2}}\right)+\ldots, & \mathcal{O}_{2}(z, \bar{z}) & =\operatorname{tr}\left(X^{K}\right), \\
\mathcal{O}_{4}(\infty) & =\operatorname{tr}\left(Z^{\frac{K}{2}} \bar{X}^{\frac{K}{2}}\right)+\ldots, & \mathcal{O}_{3}(1) & =\operatorname{tr}\left(\bar{Z}^{K}\right) .
\end{aligned}
$$

In the limit $K \rightarrow \infty$ the simplest four-point correlator factorises to a product of two identical octagons with $\ell=0$, and $\alpha=\bar{\alpha}=1$,

$$
\left\langle\mathcal{O}_{1} \mathcal{O}_{2} \mathcal{O}_{3} \mathcal{O}_{4}\right\rangle \underset{K \rightarrow \infty}{=} \frac{\left[\mathbb{O}_{0}(z, \bar{z}, 1,1)\right]^{2}}{\left(x_{12}^{2} x_{34}^{2} x_{13}^{2} x_{24}^{2}\right)^{K / 2}} .
$$

There is another class of four-point functions considered in [2], which are expressed in terms of octagons $\mathbb{O}_{\ell}$ with $\ell>0$. In the recent paper [13] the non-planar four-point correlators are expressed as polynomials of $\mathbb{O}^{2}$. It this paper we will focus exclusively on the octagon form factor $\mathbb{O}_{\ell}(z, \bar{z}, \alpha, \bar{\alpha})$.

\subsection{The octagon form factor}

Here we remind the series expansion of the octagon as a sum over mirror particles mainly with the purpose of introducing our notations which are slightly different than those of [2]. As usual we denote by $u$ the rapidity of the particles in the $2 \mathrm{~d}$ quantum field theory describing the dynamics of the single-trace operators in $\mathcal{N}=4 \mathrm{SYM}$. The dispersion relation for the physical particles $E_{a}^{2}=\frac{a^{2}}{4}+4 g^{2} \sin ^{2} \frac{p_{a}}{2}$ is parametrised by the Zhukovsky map

$$
\frac{u}{g}=x+\frac{1}{x}, \quad x(u)=\frac{u}{g}\left(\frac{1}{2}+\frac{1}{2} \sqrt{1-\frac{4 g^{2}}{u^{2}}}\right) .
$$


The momentum and the energy of the physical particles of type $a$ are given by

$$
p_{a}(u)=-i\left(\mathbb{D}^{a}-\mathbb{D}^{-a}\right) \log x, \quad E_{a}(u)=-\frac{i g}{2}\left(\mathbb{D}^{a}-\mathbb{D}^{-a}\right)\left(x-\frac{1}{x}\right),
$$

where $\mathbb{D}=e^{i \partial_{u} / 2}$ is the shift operator, to be used repeatedly in the following,

$$
\mathbb{D}: f(u) \rightarrow f(u+i / 2)
$$

For some computations this operator representation of functions with shifted arguments can be quite efficient. We will use sometimes the commonly accepted notations,

$$
f(u \pm i a / 2)=f^{[ \pm a]}(u)=\mathbb{D}^{ \pm a} f(u)=f(u)^{\mathbb{D}^{ \pm a}} .
$$

We are interested in summing over the particles in the mirror dynamics whose energy and momentum are given by

$$
\tilde{p}_{a}(u)=\frac{1}{2} g\left(\mathbb{D}^{a}+\mathbb{D}^{-a}\right)\left(x-\frac{1}{x}\right), \quad \tilde{E}_{a}(u)=\left(\mathbb{D}^{a}+\mathbb{D}^{-a}\right) \log x .
$$

We consider an octagon with four physical and four mirror edges with the corresponding BMN vacuum at each physical edge, as shown schematically in figure 1 . The octagon is obtained by gluing the hexagons $\mathcal{H}_{1}$ and $\mathcal{H}_{2}$ along the common edge $(0,0)-(\infty, \infty)$ by inserting a complete set of virtual states $\psi$ with energies $\tilde{E}_{\psi}$. A state $\psi$ contains an arbitrary number of fundamental particles and their bound states transforming in the skew-symmetric representations of $\mathfrak{p s u}(2 \mid 2) \times \mathfrak{p s u}(2 \mid 2)$. Symbolically

$$
\mathbb{O}_{\ell}=\sum_{\psi}\left\langle\mathcal{H}_{2} \mid \psi\right\rangle e^{-\tilde{E}_{\psi} \ell}\left\langle\psi \mid \mathcal{H}_{1}\right\rangle .
$$

To write the explicit expression one should bring the two hexagon operators to the canonical hexagon $\mathcal{H}$. The dependence on the cross ratios in the coordinate and flavour spaces appears through the similarity transformations $\mathcal{H}_{1} \rightarrow \mathcal{H}$ and $\mathcal{H}_{2} \rightarrow \mathcal{H}$ :

$$
\mathbb{O}_{\ell}(z, \bar{z}, \alpha, \bar{\alpha})=\sum_{\psi}\langle\mathcal{H} \mid \psi\rangle e^{-\tilde{E}_{\psi} \ell} e^{2 i \tilde{p}_{\psi} \xi} e^{i L_{\psi} \phi} e^{i R_{\psi} \theta} e^{i J_{\psi} \varphi}\langle\psi \mid \mathcal{H}\rangle .
$$

The parameters $\phi, \xi, \theta, \varphi$ conjugated to $\mathbf{L}, \boldsymbol{p}, \mathbf{R}, \mathbf{J}$ are related to the cross ratios in the Minkowski and in the flavour spaces, eq. (1.6), as

$$
\begin{aligned}
z & =e^{-\xi+i \phi}, & \bar{z} & =e^{-\xi-i \phi}, \\
\alpha & =e^{\varphi-\xi+i \theta}, & \bar{\alpha} & =e^{\varphi-\xi-i \theta} .
\end{aligned}
$$

An $n$-particle virtual state $\psi$ is completely characterised by the rapidities and the bound state numbers $\left(u_{j}, a_{j}\right)$ of the individual particles $(j=1, \ldots, n)$. Taking into account the explicit form of the hexagon form factors, one writes (1.15) as the following series of multiple integrals,

$$
\mathbb{O}_{\ell}=\sum_{n=0}^{\infty} \frac{1}{n !} \sum_{a_{1}, \ldots, a_{n} \geq 1} \int \prod_{j=1}^{n} \frac{d u_{j}}{2 \pi}(-1)^{a_{j}} \boldsymbol{\mu}_{a_{j}}\left(u_{j}, \ell, z, \bar{z}\right) W_{a_{1} \ldots a_{n}}^{\operatorname{matrix}} \prod_{j<k}^{n} \tilde{H}_{a_{j}, a_{k}}\left(u_{j}, u_{k}\right) .
$$

The three factors in the integrand/summand are defined as follows. 
- The local integration measure

$$
\boldsymbol{\mu}_{a}(u)=\tilde{\mu}_{a}(u) e^{-\tilde{E}_{a}(u) \ell} e^{2 i \xi \tilde{p}_{a}(u)}
$$

contains the intrinsic measure coming from the canonical hexagons

$$
\tilde{\mu}_{a}(u)=\frac{x^{[+a]}-x^{[-a]}}{x^{[+a]} x^{[-a]}-1} \times \frac{1}{i g} \prod_{\varepsilon= \pm} \frac{1}{\left(x^{[\varepsilon a]}-1 / x^{[\varepsilon a]}\right)}
$$

as well as factors depending on $\xi=-\frac{1}{2} \log z \bar{z}$ and the bridge length $\ell$.

- The symmetric bilocal factors $\tilde{H}_{a b}(u, v)$ are produced by the diagonal part of the hexagon weights in mirror-mirror kinematics

$$
\tilde{H}_{a b}(u, v)=\prod_{\varepsilon, \delta= \pm} \frac{x^{[\varepsilon a]}-y^{[\delta b]}}{x^{[\varepsilon a]} y^{[\delta b]}-1},
$$

where $x=x(u)$ and $y=x(v)$. The bi-local weights can be written with the help of the shift operator (1.11) as

$$
\tilde{H}_{a b}(u, v)=K(u, v)^{\left(\mathbb{D}_{u}^{a}+\mathbb{D}_{u}^{-a}\right)\left(\mathbb{D}_{v}^{b}+\mathbb{D}_{v}^{-b}\right)}, \quad K(u, v)=\frac{x-y}{x y-1} .
$$

- The factor $W_{a_{1} \ldots a_{n}}^{\text {matrix }}$ accounts for the matrix part. The contraction of the flavour indices in the matrix factor can be done using the unitarity of the $\mathfrak{p s u}(2 \mid 2)$ S-matrix and the result is expressed in terms of the traces (transfer matrices), which in absence of physical excitations are simply the $\mathfrak{p s u}(2 \mid 2)$ characters. ${ }^{4}$ The characters are expressed in terms of the variables $\phi, \varphi, \alpha$ parametrizing the cross sections in the coordinate and in the flavour space. As suggested in $[8,10]$ and elaborated upon in [2], one should consider two ways of dressing the mirror basis with $\mathcal{Z}$ markers and then take the average between the two choices. The matrix part takes the form of a sum of two terms

$$
W_{a_{1} \ldots a_{n}}^{\text {matrix }}=\frac{1}{2}\left(\prod_{j=1}^{n} \chi_{a_{j}}^{+}+\prod_{j=1}^{n} \chi_{a_{j}}^{-}\right)
$$

where $\chi_{a}^{ \pm}$are the characters of the antisymmetric representations of $\mathfrak{p s u}(2 \mid 2),[8,10]$ (cf eq. (47) of [8])

$$
\operatorname{tr}_{a}\left[(-1)^{F} e^{\varphi \mathbf{J}+i \phi \tilde{\mathbf{L}}+i \theta \tilde{\mathbf{R}}}\right]=\chi_{a}^{+}+\chi_{a}^{-}, \quad \chi_{a}^{ \pm}=(-1)^{a} \lambda^{ \pm} \frac{\sin (a \phi)}{\sin \phi},
$$

where the dependence on $\varphi$ and $\theta$ is carried out by the factor ${ }^{5}$

$$
\lambda^{ \pm}(\phi, \varphi, \theta)=2[\cos \phi-\cosh (\varphi \pm i \theta)] .
$$

\footnotetext{
${ }^{4}$ This is true only if the integrand does not contain singularities as e.g. double poles.

${ }^{5}$ The factor $\lambda^{ \pm}$is related to $\mathcal{X}^{ \pm}$in $[2]$ by $\mathcal{X}^{+}=-\frac{(z-\alpha)(\bar{z}-\alpha)}{\alpha}=\lambda^{+} e^{-\xi} ; \mathcal{X}^{-}=-\frac{(z-\bar{\alpha})(\bar{z}-\bar{\alpha})}{\bar{\alpha}}=\lambda^{-} e^{-\xi}$.
} 
This form of the $\mathfrak{p s u}(2 \mid 2)$ characters follows from the generating function

$$
\begin{aligned}
\mathcal{W}_{ \pm}(t) & =\sum_{a=0}^{\infty}(-1)^{a} \chi_{a}^{ \pm} e^{a t}=\frac{\left(1-e^{ \pm \varphi+i \theta} e^{t}\right)\left(1-e^{\mp \varphi-i \theta} e^{t}\right)}{\left(1-e^{i \phi} e^{t}\right)\left(1-e^{-i \phi} e^{t}\right)} \\
& =1+\frac{\lambda^{ \pm}}{2(\cosh t-\cos \phi)}
\end{aligned}
$$

The final expression of the series expansion for the octagon takes a form resembling a Coulomb gas of dipole charges

$$
\mathbb{O}_{\ell}(g, z, \bar{z}, \alpha, \bar{\alpha})=1+\sum_{n=1}^{\infty} \mathcal{X}_{n}(\phi, \varphi, \alpha) \mathcal{I}_{n, \ell}(z, \bar{z})
$$

with

$$
\begin{aligned}
\mathcal{I}_{n, \ell}(z, \bar{z}) & =\frac{1}{n !} e^{n \xi} \sum_{a_{1}, \ldots, a_{n} \geq 1} \prod_{j=1}^{n} \frac{\sin \left(a_{j} \phi\right)}{\sin \phi} \int \prod_{j=1}^{n} \frac{d u_{j}}{2 \pi} \boldsymbol{\mu}_{a_{j}}\left(u_{j}\right) \prod_{j<k} \tilde{H}_{a_{j}, a_{k}}\left(u_{j}, u_{k}\right), \\
\mathcal{X}_{n} & =\frac{\left(\lambda^{+}\right)^{n}+\left(\lambda^{-}\right)^{n}}{2} e^{-n \xi}
\end{aligned}
$$

The multiple contour integrals were evaluated by residues order by order up to $n=4$ in [2]. The perturbative result for the octagon obtained in [2] matched the five loops results in [23] obtained previously using the conformal symmetry, the hidden dual conformal symmetry and analytic bootstrap conditions. As explained before, it allows to extend these results to any loop order.

\subsection{Summary of the results}

\subsubsection{The octagon ${ }^{2}$ as a determinant}

Based on the representation of the series (1.26) as Fredholm pfaffian, explained in section 2.1, we give an explicit formula for the octagon in terms of the square root of the determinant,

$$
\mathbb{O}_{\ell}(z, \bar{z}, \alpha, \bar{\alpha})=\frac{1}{2} \sum_{ \pm} \sqrt{\operatorname{Det}\left[\mathbf{I}-\lambda^{ \pm} \mathbf{C K}\right]}
$$

The matrices $\mathbf{I}, \mathbf{C}$ and $\mathbf{K}$ are semi-infinite matrices of the type $\mathbf{M}=\left\{\mathrm{M}_{m, n}\right\}_{m, n=0}^{\infty}$. The matrices $\mathbf{I}$ and $\mathbf{C}$ are standard,

$$
\mathrm{I}_{m, n}=\delta_{m, n}, \quad \mathrm{C}_{n m}=\delta_{n+1, m}-\delta_{n, m+1}, \quad m, n \geq 0,
$$

while the matrix $\mathbf{K}$ depends on the gauge coupling $g$ and the cross ratios in the coordinate space through the angle $\phi$ and the parameter $\xi$ defined in (1.16). Its matrix elements $\mathrm{K}_{n m}$ are given for any coupling $g$ by a non-singular integral of a product of two Bessel functions,

$$
\mathrm{K}_{m n}=\frac{g}{2 i} \int_{|\xi|}^{\infty} d t \frac{\left(i \sqrt{\frac{t+\xi}{t-\xi}}\right)^{m-n}-\left(i \sqrt{\frac{t+\xi}{t-\xi}}\right)^{n-m}}{\cos \phi-\cosh t} J_{m+\ell}\left(2 g \sqrt{t^{2}-\xi^{2}}\right) J_{n+\ell}\left(2 g \sqrt{t^{2}-\xi^{2}}\right),
$$


while the dependence on the cross ratios for the polarisations occurs through the factors $\lambda^{ \pm}$defined in (1.24). According to (1.29) the octagon is expressed in terms of the moments of the matrix CK,

$$
\begin{aligned}
\mathbb{O}_{\ell} & =\frac{1}{2} e^{\mathcal{S}^{+}}+\frac{1}{2} e^{\mathcal{S}^{-}}, \\
\mathcal{S}^{ \pm} & =-\frac{1}{2} \sum_{n=1}^{\infty} \frac{\left(\lambda^{ \pm}\right)^{n}}{n} \operatorname{tr}\left[(\mathbf{C K})^{n}\right] .
\end{aligned}
$$

The $n$-th moment gives the contribution of a connected cluster of $n$ virtual particles. Formula (1.32) provides an explicit expression for the Coronado integrals in (1.26) in terms of the moments of the matrix $\mathbf{C K}$

$$
n \mathcal{I}_{n, \ell}=-\frac{1}{2} \sum_{k=0}^{n-1} \mathcal{I}_{k, \ell} \operatorname{tr}\left[\left(e^{\xi} \mathbf{C K}\right)^{n-k}\right], n \geq 1, \mathcal{I}_{0, \ell}=1 .
$$

In the main body of the paper we also represent (1.29) as the pfaffian of a semi-infinite matrix.

\subsubsection{Perturbative expansion}

The expression (1.29) is true for any coupling $g$. If one is interested in the perturbative expansion at weak coupling, the formula can be made more explicit. Within the perturbative expansion the octagon in (1.29) becomes a determinant and the formula for the octagon reads

$$
\mathbb{O}_{\ell}=\frac{1}{2} \sum_{ \pm} \operatorname{det}\left(1+\mathcal{X}^{ \pm} \mathcal{R}\right)
$$

where $\mathcal{X}^{ \pm}=\lambda^{ \pm} e^{-\xi}$ and the elements of the matrix $\mathcal{R}=\left\{\mathcal{R}_{k, j}\right\}_{k, j \geq 0}$ are infinite linear combinations of the ladder integrals (1.3),

$$
\mathcal{R}_{k j}=\sum_{p=\max (k+j+\ell, 1+j+\ell)}^{\infty}(-1)^{p-\ell} \frac{\Gamma(2 p)\left(2 p(2 k+\ell)-\delta_{k, 0}(p-j)(p+j+\ell)\right)}{\prod_{ \pm} \Gamma(p \pm(k-j)+1) \Gamma(p \pm(k+j+\ell)+1)} f_{p}(z, \bar{z}) g^{2 p}
$$

where $f_{p}$ are given in (1.3). In actual computations it is convenient to truncate the semiinfinite matrix $\mathcal{R}$ to a $N \times N$ matrix $\mathcal{R}_{N \times N}=\left\{\mathcal{R}_{k, j}\right\}_{0 \leq k, j \leq N-1}$. Such a truncation reproduces the perturbative expansion of the octagon to loop order $2 N-1$,

$$
\mathbb{O}_{\ell}=\frac{1}{2} \sum_{ \pm} \operatorname{det}\left(1+\mathcal{X}^{ \pm} \mathcal{R}\right)_{N \times N}+o\left(g^{4 N+2 \ell}\right) .
$$

For example, the truncation to a $3 \times 3$ matrix gives the perturbative expansion up to $o\left(g^{12}\right)$,

$$
\begin{aligned}
\mathbb{O}_{\ell=0}= & \frac{1}{2} \operatorname{det}\left(1+\mathcal{X}^{+} \mathcal{R}\right)_{3 \times 3}+\frac{1}{2} \operatorname{det}\left(1+\mathcal{X}^{-} \mathcal{R}\right)_{3 \times 3}+o\left(g^{12}\right) \\
= & 1+\mathcal{X}_{1}\left(f_{1} g^{2}-f_{2} g^{4}+\frac{1}{2} f_{3} g^{6}-\frac{5}{36} f_{4} g^{8}+\frac{7}{288} f_{5} g^{10}\right) \\
& +\mathcal{X}_{2}\left(\frac{1}{12}\left(f_{1} f_{3}-f_{2}^{2}\right) g^{8}-\frac{1}{24}\left(f_{1} f_{4}-f_{2} f_{3}\right) g^{10}\right)+o\left(g^{12}\right) .
\end{aligned}
$$


The nine loop result presented in [2] is reproduced by truncating to a $5 \times 5$ matrix. To compare with [2] one should take $f_{n}=n !(n-1) ! F_{n}$.

\subsubsection{The null-square limit}

Here we comment shortly the null-square limit when the intervals between two subsequent operators in figure 1 become light-like, $x_{12}^{2}, x_{13}^{2}, x_{34}^{2}, x_{42}^{2} \rightarrow 0$, or $z \rightarrow 0,1 / \bar{z} \rightarrow 0$. In [18] it was conjectured that the logarithm of the octagon enjoys the following simple asymptotics,

$$
\begin{aligned}
\lim _{\substack{z \rightarrow 0 \\
z \rightarrow \infty}} \log \mathbb{O}= & -\tilde{\Gamma}(g)(\log (-z)+\log (-1 / \bar{z}))^{2} \\
& +\frac{1}{2} g^{2}\left([\log (-z)]^{2}+[\log (-1 / \bar{z})]^{2}\right)+\text { constant. }
\end{aligned}
$$

The coefficient $\tilde{\Gamma}$ is somehow similar to the cusp anomalous dimension.

We were able to reproduce this asymptotics in the weak coupling limit from the determinant formula. In the null-square limit $\mathcal{X}_{n}=\bar{z}^{n}$ and the determinant formula (1.34) gives for the logarithm of the octagon

$$
\log \mathbb{O}=\operatorname{Tr} \log (1+\bar{z} \boldsymbol{\mathcal { R }})=\bar{z} \operatorname{tr} \mathcal{R}-\frac{\bar{z}^{2}}{2} \operatorname{tr} \boldsymbol{\mathcal { R }}^{2}+\frac{\bar{z}^{3}}{3} \operatorname{tr} \boldsymbol{\mathcal { R }}^{3}-\ldots
$$

The matrix elements of $\mathcal{R}$ simplify in this limit and for the determinant we obtain, after massive cancellations, the asymptotics (1.37). We checked that the perturbative expansion of $\tilde{\Gamma}(g)$ is in agreement with [18]. To compute $\tilde{\Gamma}(g)$ to $N$ loops it is sufficient to keep the first $N$ terms in the series (1.38) and truncate $\mathcal{R}$ to an $N \times N$ matrix. For example, if we take $N=10$, we obtain the nine-loop result

$$
\begin{aligned}
\tilde{\Gamma}(g)= & \frac{1}{2} g^{2}-\frac{1}{6} \pi^{2} g^{4}+\frac{8}{45} \pi^{4} g^{6}-\frac{68}{315} \pi^{6} g^{8}+\frac{3968}{14175} \pi^{8} g^{10}-\frac{176896}{467775} \pi^{10} g^{12} \\
& +\frac{22368256}{42567525} \pi^{12} g^{14}-\frac{475939328}{638512875} \pi^{14} g^{16}+\frac{104932671488}{97692469875} \pi^{16} g^{18}+o\left(g^{20}\right) .
\end{aligned}
$$

\section{The octagon as a pfaffian}

\subsection{The octagon as a Fredholm pfaffian}

It has been pointed out in [14] that in some simple configurations the sum over the virtual particles can be recast in the form of a Fredholm pfaffian [24]. In this subsection we will develop the pfaffian representation in full detail. We first remind the definition of a Fredholm pfaffian in general.

Let $\boldsymbol{K}\left(u_{1}, u_{2}\right)$ be a $2 \times 2$ antisymmetric matrix kernel of trace class defined on $\mathbb{R}$,

$$
\boldsymbol{K}\left(u_{1}, u_{2}\right)=\left(\begin{array}{ll}
K^{++}\left(u_{1}, u_{2}\right) & K^{+-}\left(u_{1}, u_{2}\right) \\
K^{-+}\left(u_{1}, u_{2}\right) & K^{--}\left(u_{1}, u_{2}\right)
\end{array}\right), \quad K^{\varepsilon_{1} \varepsilon_{2}}\left(u_{1}, u_{2}\right)=-K^{\varepsilon_{2} \varepsilon_{1}}\left(u_{2}, u_{1}\right) .
$$

Denote by $\boldsymbol{J}$ the standard $2 \times 2$ antisymmetric matrix kernel

$$
\boldsymbol{J}=\left(\begin{array}{cc}
0 & 1 \\
-1 & 0
\end{array}\right) \delta\left(u_{1}-u_{2}\right)
$$


The Fredholm pfaffian $\operatorname{Pf}[\boldsymbol{J}+\boldsymbol{K}]$ is defined as the series of multiple integrals with some integration measure $d \mu(u)$

$$
\operatorname{Pf}[\boldsymbol{J}+\boldsymbol{K}]=\sum_{n=0}^{\infty} \frac{1}{n !} \int_{\mathbb{R}} \prod_{j=1}^{n} d \mu\left(u_{j}\right) \operatorname{Pf}\left[\boldsymbol{K}_{n}\left(u_{1}, \ldots, u_{n}\right)\right],
$$

where $\boldsymbol{K}_{n}\left(u_{1}, \ldots, u_{n}\right)$ is the $2 n \times 2 n$ antisymmetric matrix

$$
\boldsymbol{K}_{n}\left(u_{1}, \ldots, u_{n}\right)=\left[\boldsymbol{K}\left(u_{j}, u_{k}\right)\right]_{1 \leq j, k \leq n}
$$

where $\boldsymbol{K}(u, v)$ is the $2 \times 2$ matrix (2.1). The relation with the Fredholm determinant is

$$
\operatorname{Pf}[\boldsymbol{J}+\boldsymbol{K}]=\sqrt{\operatorname{det}[\boldsymbol{I}-\boldsymbol{J} \boldsymbol{K}]}
$$

where $\boldsymbol{I}$ is the $2 \times 2$ matrix kernel of the identity operator.

Now let us focus on the series (1.26). Using the pfaffian version of the Cauchy identity

$$
\prod_{j<k}^{2 n} \frac{x_{j}-x_{k}}{x_{j} x_{k}-1}=\operatorname{Pf}\left(\left[\frac{x_{j}-x_{k}}{x_{j} x_{k}-1}\right]_{i, j=1}^{2 n}\right)
$$

we can write the product of the bi-local weights (1.21) as a pfaffian. In order to illustrate how the series is assembled into a Fredholm pfaffian, let us first neglect the bound states with $a \geq 2$ and consider only the sum over fundamental particles $(a=1)$. It is quite obvious that the grand canonical sum of virtual particles is the expansion of a Fredholm pfaffian of the skew-symmetric kernel

$$
K^{\varepsilon_{1}, \varepsilon_{2}}\left(u_{1}, u_{2}\right)=K\left(u_{1}+i \frac{\varepsilon_{1}}{2}, u_{2}+i \frac{\varepsilon_{2}}{2}\right), \quad \varepsilon_{1}, \varepsilon_{2}= \pm .
$$

which is obtained by shifting with $\pm i / 2$ the arguments of the kernel (1.21),

$$
K(u, v)=\frac{x(u)-x(v)}{x(u) x(v)-1} .
$$

In order to formulate the exact statement we need to specify the measure.

The $n$-particle pfaffians (2.3) then give the product of the bi-local factors in the integrand in (1.26) times pieces of the local measure. For that we will compare the terms with $n=1$ and $n=2$ in the expansion (2.3) with the contributions of one- and two-particle states to the octagon.

- For $n=1$

$$
\boldsymbol{K}_{1}(u)=\left(\begin{array}{cc}
0 & \frac{x^{+}-x^{-}}{x^{-} x^{+}-1} \\
\frac{x^{-}-x^{+}}{x^{-} x^{+}-1} & 0
\end{array}\right)
$$

and the pfaffian gives the first factor in the intrinsic measure (1.19) with $a=1$,

$$
\operatorname{Pf}(u) \equiv \operatorname{Pf}\left[\boldsymbol{K}_{1}(u)\right]=\frac{x^{+}-x^{-}}{x^{+} x^{-}-1} .
$$


- For $n=2$

$$
\boldsymbol{K}_{2}\left(u_{1}, u_{2}\right)=\left(\begin{array}{cccc}
0 & \frac{x^{+}-x^{-}}{x^{-} x^{+}-1} & \frac{x^{+}-y^{+}}{x^{+} y^{+}-1} & \frac{x^{+}-y^{-}}{x^{+} y^{-}-1} \\
\frac{x^{-}-x^{+}}{x^{-} x^{+}-1} & 0 & \frac{x^{-}-y^{+}}{x^{-} y^{+}-1} & \frac{x^{-}-y^{-}}{x^{-} y^{-}-1} \\
\frac{y^{+}-x^{+}}{x^{+} y^{+}-1} & \frac{y^{+}-x^{-}}{x^{-} y^{+}-1} & 0 & \frac{y^{+}-y^{-}}{y^{-} y^{+}-1} \\
\frac{y^{-}-x^{+}}{x^{+} y^{-}-1} & \frac{y^{-}-x^{-}}{x^{-} y^{-}-1} & \frac{y^{-}-y^{+}}{y^{-} y^{+}-1} & 0
\end{array}\right), \quad x=x\left(u_{1}\right), y=y\left(u_{2}\right) .
$$

It is identified with the matrix in (2.6) for $n=2$, with $x_{1}=x^{+}, x_{2}=x^{-}, x_{3}=y^{+}$, $x_{4}=y^{-}$.

The pfaffian gives the bilocal weight $\tilde{H}_{11}\left(u_{1}, u_{2}\right)$ in the integrand for the octagon, eq. (1.20), times the factors $\operatorname{Pf}\left(u_{1}\right) \operatorname{Pf}\left(u_{2}\right)$,

$$
\operatorname{Pf}\left(u_{1}, u_{2}\right) \equiv \operatorname{Pf}\left[\boldsymbol{K}_{2}\left(u_{1}, u_{2}\right)\right]=\operatorname{Pf}\left(u_{1}\right) \operatorname{Pf}\left(u_{2}\right) \tilde{H}_{11}\left(u_{1}, u_{2}\right) .
$$

- For general $n$ the pfaffian of $\boldsymbol{K}_{n}$ is given by the product

$$
\operatorname{Pf}\left(u_{1}, \ldots, u_{n}\right) \equiv \operatorname{Pf}\left[\boldsymbol{K}_{n}\left(u_{1}, \ldots, u_{n}\right)\right]=\prod_{j=1}^{n} \operatorname{Pf}\left(u_{j}\right) \prod_{j<k}^{n} \tilde{H}_{11}\left(u_{j}, u_{k}\right) .
$$

Hence the measure $d \mu(u)$ is given by the measure (1.19) stripped from the first factor.

Now let us consider the complete sum. In order to include the bound states, we have upgraded the continuous variable $u \in \mathbb{R}$ to a pair $(u, a) \in \mathbb{R} \times \mathbb{N}$. Instead of the $2 \times 2$ matrix kernel $\boldsymbol{K}\left(u_{1}, u_{2}\right)$ defined on $\mathbb{R}^{\times 2}$, eq. (2.7), we have to deal with the $2 \times 2$ matrix kernel $\boldsymbol{K}\left(u_{1}, a_{1} ; a_{2}, u_{2}\right)$ defined on $(\mathbb{R} \times \mathbb{N})^{\times 2}$ and having matrix elements

$$
K^{\varepsilon_{1}, \varepsilon_{2}}\left(u_{1}, a_{1} ; u_{2}, a_{2}\right)=K\left(u_{1}^{\left[\varepsilon_{1} a_{1}\right]}, u_{2}^{\left[\varepsilon_{2} a_{2}\right]}\right), \quad \varepsilon_{1,2}= \pm .
$$

The functions $x^{[ \pm a]}=x(u \pm i a / 2)$ are taken in the first sheet, where $|x(u)|>1$. The pfaffians for one- and two-particle states are

$$
\begin{gathered}
\operatorname{Pf}(u, a) \equiv \operatorname{Pf}\left[\boldsymbol{K}_{1}(u, a)\right]=\frac{x^{[+a]}-x^{[-a]}}{x^{[+a]} x^{[-a]}-1} \\
\operatorname{Pf}(u, a ; v, b) \equiv \operatorname{Pf}\left[\boldsymbol{K}_{2}(u, a ; v, b)\right]=\operatorname{Pf}(u, a) \operatorname{Pf}(v, b) H_{a b}(u, v) .
\end{gathered}
$$

The pfaffian for a single particle gives a piece of the measure (1.19). Let us denote the remaining piece by $\omega(u, a)$ :

$$
\tilde{\mu}_{a}(u)=\operatorname{Pf}(u, a) \omega(u, a), \quad \omega(u, a)=\frac{1}{i g} \prod_{\varepsilon= \pm} \frac{1}{\left(x^{[\varepsilon a]}-1 / x^{[\varepsilon a]}\right)} .
$$

Now we are able to formulate the exact claim. The expansion of the octagon (1.26) as a sum of two Fredholm pfaffians

$$
\mathbb{O}_{\ell}=\frac{1}{2} \sum_{ \pm} \sum_{n=0}^{\infty} \frac{\left(\lambda^{ \pm}\right)^{n}}{n !} \sum_{a_{1}, \ldots, a_{n} \geq 1} \int \prod_{j=1}^{n} d \mu\left(u_{j}, a_{j}\right) \operatorname{Pf}\left[\boldsymbol{K}_{n}\left(u_{1}, a_{1} ; \ldots ; u_{n}, a_{n}\right)\right]
$$


with the integration measure given by

$$
d \mu(u, a)=\frac{\sin (a \phi)}{\sin \phi} \frac{d u}{2 \pi} \omega(u, a) e^{-\tilde{E}_{a}(u) \ell} e^{2 i \xi \tilde{p}_{a}(u)} .
$$

In view of the subsequent analysis, it will be important that the integration measure (2.18) can be written in a factorised form

$$
d \mu(u, a)=\frac{1}{i g} \frac{\sin (a \phi)}{\sin \phi} \frac{d u}{2 \pi} \Omega_{\ell}(u+i a / 2) \Omega_{\ell}(u-i a / 2),
$$

with the function $\Omega_{\ell}(u)$ defined as

$$
\Omega_{\ell}(u) \equiv \frac{e^{i g \xi[x(u)-1 / x(u)]}}{x(u)-1 / x(u)} x(u)^{-\ell} .
$$

To summarise, the expansion (1.26) of the octagon sums up to a Fredholm pfaffian which by (2.5) is a square root of a Fredholm determinant,

$$
\begin{aligned}
\mathbb{O}_{\ell}(z, \bar{z}, \alpha, \bar{\alpha}) & =\frac{1}{2} \sum_{ \pm} \operatorname{Pf}\left(\boldsymbol{J}+\lambda^{ \pm} \boldsymbol{K}\right) \\
& =\frac{1}{2} \sum_{ \pm} \sqrt{\operatorname{Det}\left(\boldsymbol{I}-\lambda^{ \pm} \boldsymbol{J} \boldsymbol{K}\right)},
\end{aligned}
$$

with the matrix elements of the kernel $\boldsymbol{K}$ defined in (2.14) and the integration measure given by $(2.19)-(2.20)$.

\subsection{Summing up the bound states}

In order to perform the sum over the bound states it is more convenient to start with the determinant representation in the second line of (2.21),

$$
\mathbb{O}_{\ell}(z, \bar{z}, \alpha, \bar{\alpha})=\frac{1}{2} e^{\mathcal{S}^{+}}+\frac{1}{2} e^{\mathcal{S}^{-}}
$$

with

$$
\begin{aligned}
\mathcal{S}^{ \pm}= & \frac{1}{2} \operatorname{Tr} \log \left(\boldsymbol{I}-\lambda^{ \pm} \boldsymbol{J} \boldsymbol{K}\right) \\
= & -\frac{1}{2} \sum_{n=1}^{\infty} \frac{\left(\lambda^{ \pm}\right)^{n}}{n} \frac{1}{(i g)^{n}} \sum_{a_{1}, \ldots, a_{n} \geq 1} \sum_{\varepsilon_{1}, \ldots, \varepsilon_{n}= \pm} \prod_{j=1}^{n} \frac{\sin \left(a_{j} \phi\right)}{\sin \phi} \\
& \times \int_{\mathbb{R}} \prod_{j=1}^{n} \frac{d u_{j}}{2 \pi} \Omega_{\ell}^{\left[-\varepsilon_{j} a_{j}\right]}\left(u_{j}\right) \Omega_{\ell}^{\left[+\varepsilon_{j} a_{j}\right]}\left(u_{j}\right) \\
& \times \varepsilon_{1} K\left(u_{1}^{\left[-\varepsilon_{1} a_{1}\right]}, u_{2}^{\left[\varepsilon_{2} a_{2}\right]}\right) \varepsilon_{2} K\left(u_{2}^{\left[-\varepsilon_{2} a_{2}\right]}, u_{3}^{\left[\varepsilon_{3} a_{3}\right]}\right) \ldots \varepsilon_{n} K\left(u_{n}^{\left[-\varepsilon_{n} a_{n}\right]}, u_{1}^{\left[\varepsilon_{1} a_{1}\right]}\right) .
\end{aligned}
$$

The factors $\varepsilon_{j}$ come from $J^{\varepsilon,-\varepsilon}=\varepsilon, J^{\varepsilon, \varepsilon}=0, \varepsilon= \pm$.

We will take advantage of the fact that the elements of the matrix kernel (2.14) are obtained by shifting the arguments of the scalar kernel (2.8). This will allow us to replace 
the sum over the labels $a_{j}$ by an appropriate difference operator. This is not unrelated to the fact that the sum over the $\mathfrak{p} \mathfrak{s u}(2 \mid 2)$ characters gives the generating function (1.25). We will obtain the same generating function, but with $t$ replaced by $i \partial_{u}$.

First we notice that the integrand is analytic in the strip $-a_{j} / 2<\operatorname{Im}\left(u_{j}\right)<a_{j} / 2$, which allows us to displace the contour for $u_{j}$ by $-i \varepsilon_{j}\left(a_{j}-\epsilon\right) / 2$. Here $\epsilon$ is a small positive quantity which will be sent to 0 in the final expression. We will keep it finite during the computation because it indicates whether we are above or below the real axis on which $x(u)$ has a cut. The displacement of the contours can be compensated by shifts of the variables $u_{j} \rightarrow u_{j}+i \varepsilon_{j} a_{j} / 2$. As a result of this manipulation the integrand for the $n$-th term takes the form

$$
\begin{aligned}
& \int_{\mathbb{R}} \frac{d u_{j}}{2 \pi} \Omega^{\left[-i \epsilon \varepsilon_{j}\right]}\left(u_{j}\right) \Omega^{\left[2 \varepsilon_{j} a_{j}\right]}\left(u_{j}\right) \\
& \times \varepsilon_{1} K\left(u_{1}^{\left[-i \epsilon \varepsilon_{1}\right]}, u_{2}^{\left[2 \varepsilon_{2} a_{2}\right]}\right) \varepsilon_{2} K\left(u_{2}^{\left[-i \epsilon \varepsilon_{2}\right]}, u_{3}^{\left[2 \varepsilon_{3} a_{3}\right]}\right) \ldots \varepsilon_{n} K\left(u_{n}^{\left[-i \epsilon \varepsilon_{n}\right]}, u_{1}^{\left[2 \varepsilon_{1} a_{1}\right]}\right) \\
& =\int_{\mathbb{R}+i \epsilon \varepsilon_{j}} \frac{d u_{j}}{2 \pi} \varepsilon_{1} \hat{K}\left(u_{1}^{\left[-i \epsilon \varepsilon_{1}\right]}, u_{2}^{\left[2 \varepsilon_{2} a_{2}\right]}\right) \varepsilon_{2} \hat{K}\left(u_{2}^{\left[-i \epsilon \varepsilon_{2}\right]}, u_{3}^{\left[2 \varepsilon_{3} a_{3}\right]}\right) \ldots \varepsilon_{n} \hat{K}\left(u_{n}^{\left[-i \epsilon \varepsilon_{n}\right]}, u_{1}^{\left[2 \varepsilon_{1} a_{1}\right]}\right),
\end{aligned}
$$

where the non-flat part of the measure is absorbed in the kernel by dressing it with two factors (2.20),

$$
K(u, v) \rightarrow \hat{K}(u, v)=\Omega_{\ell}(u) K(u, v) \Omega_{\ell}(v) .
$$

The advantage of this rewriting is that now the multiple sums over the bound state labels $a_{1}, \ldots, a_{n}$ in the cyclic integral decouples. The sum over each label $a_{j}$ can be most easily performed by introducing the shift operator $\mathbb{D}_{u_{j}}$ with the result being a difference operator acting on $\hat{K}\left(u_{j-1}, u_{j}\right)$.

In this way we traded the multiple sum over the bound state labels for a more complicated $2 \times 2$ matrix kernel $\mathbb{K}=\left\{\mathbb{K}^{\epsilon, \delta}\right\}_{\epsilon, \delta= \pm}$ whose matrix elements are

$$
\begin{aligned}
\mathbb{K}^{\varepsilon, \delta}(u, v) & \stackrel{\text { def }}{=} \frac{1}{i g} \sum_{a \geq 1} \frac{e^{i a \phi}-e^{-i a \phi}}{e^{i \phi}-e^{-i \phi}} \hat{K}(u+i \varepsilon \epsilon, v+i \delta a) \\
& =-\frac{1}{i g} \frac{1}{2 \cos \phi-2 \cos \left(\partial_{v}\right)} \hat{K}(u+i \varepsilon \epsilon, v+i \delta \epsilon) .
\end{aligned}
$$

The action of the operator function in the last line is well defined for any $\epsilon>0$. Returning to the series (2.23), we write it as the logarithm of a Fredholm determinant of the $2 \times 2$ matrix kernel $\boldsymbol{J} \mathbb{K}$

$$
\begin{aligned}
\mathcal{S}^{ \pm} & =-\sum_{n=1}^{\infty} \frac{\left(\lambda^{ \pm}\right)^{n}}{2 n} \sum_{\varepsilon_{1}, \ldots, \varepsilon_{n}= \pm} \int_{\mathbb{R}} \prod_{j=1}^{n} \frac{d u_{j}}{2 \pi} \prod_{j=1}^{n} \varepsilon_{j} \mathbb{K}^{-\varepsilon_{j}, \varepsilon_{j+1}}\left(u_{j}, u_{j+1}\right) \\
& =\frac{1}{2} \operatorname{Tr} \log \left(\boldsymbol{I}-\lambda^{ \pm} \boldsymbol{J} \mathbb{K}\right),
\end{aligned}
$$

and respectively for the octagon

$$
\mathbb{O}_{\ell}(z, \bar{z}, \alpha, \bar{\alpha})=\frac{1}{2} \sum_{ \pm} \sqrt{\operatorname{Det}\left[\boldsymbol{I}-\lambda^{ \pm} \boldsymbol{J} \mathbb{K}\right]}
$$


The corresponding pfaffian representation is

$$
\mathbb{O}_{\ell}(z, \bar{z}, \alpha, \bar{\alpha})=\frac{1}{2} \sum_{ \pm} \operatorname{Pf}\left[\boldsymbol{J}+\lambda^{ \pm} \mathbb{K}\right]
$$

\subsection{From Fredholm kernel to a semi-infinite matrix}

Our next goal is to perform the multiple cyclic integrations. This can be done by breaking the cyclic integral into a sum of products of independent integrals. The formula we are going to obtain is an infinite-dimensional version of the pfaffian integration theorem $[16,17]$. The idea is simple. We will expand the scalar kernel (2.8) in a complete set of harmonic functions on the Zhukovsky Riemann surface, which will allow us to disentangle the multiple integrals in (2.27) and represent the Fredholm pfaffian as the Pfafian of a semi-infinite skewsymmetric matrix.

The mode expansion of the scalar kernel $K$ reads for $|x|>1$ and $|y|>1$

$$
K(u, v)=\frac{x-y}{x y-1}=\sum_{m, n=0}^{\infty} x^{-n} \mathrm{C}_{n m} y^{-m},
$$

where

$$
\mathrm{C}_{n m}=\delta_{n+1, m}-\delta_{n, m+1}, \quad m, n \geq 0 .
$$

We will denote by $\mathbf{C}$ the semi-infinite antisymmetric matrix with elements $\mathbf{C}_{n m}$. Assuming that the variables $u$ and $v$ are in the first sheet $(|x|>1,|y|>1)$, we represent the dressed kernel $\hat{K}$, eq. (2.25), as a double series

$$
\hat{K}(u, v)=\sum_{n, m \geq 0} \mathrm{C}_{n m} \Omega_{\ell+n}(u) \Omega_{\ell+m}(v),
$$

with the functions $\Omega_{j}(u)$ defined by (2.20). The kernel (2.26) expands in a similar way,

$$
\mathbb{K}^{\varepsilon \delta}(u, v)=-\frac{1}{i g} \sum_{m, n \geq 0} \Omega_{\ell+n}(u+\varepsilon i \epsilon) \frac{\mathrm{C}_{n m}}{2 \cos \phi-2 \cos \partial_{v}} \Omega_{\ell+m}(v+\delta i \epsilon) .
$$

The operator sandwiched between the two $\Omega$-functions is obtained as in (2.26). The only subtlety is that when $\delta=-$, it represents a power series of $\mathbb{D}^{-1}$ which shifts by $-i / 2$.

After expanding in this way the kernels in the $n$-th term of the series (2.27), the chain of $n$ entangled integrals decouple into a sum of products of $n$ simple integrals. The exponents $\mathcal{S}^{ \pm}$take the form

$$
\mathcal{S}^{ \pm}=-\frac{1}{2} \sum_{n=1}^{\infty} \frac{\left(\lambda^{ \pm}\right)^{n}}{n} \operatorname{tr}(\mathbf{C K})^{n}
$$

where $\mathbf{K}$ is a semi-infinite skew-symmetric matrix with matrix elements $(m, n \geq 0)$

$$
\begin{aligned}
\mathrm{K}_{m n} & =\mathcal{P}_{m n}-\mathcal{P}_{n m} \\
\mathcal{P}_{m n} & =-\frac{1}{2 i g} \int \frac{d u}{2 \pi} \Omega_{\ell+n}(u-i 0) \frac{1}{\cos \phi-\cos \partial_{u}} \Omega_{\ell+m}(u+i 0) .
\end{aligned}
$$


The above procedure is nothing but a change of the basis for the operator representing the Fredholm kernel. The determinantal representation (2.28) takes in the new discrete basis the form

$$
\mathbb{O}_{\ell}(z, \bar{z}, \alpha, \bar{\alpha})=\frac{1}{2} \sum_{ \pm} \sqrt{\operatorname{Det}\left[1-\lambda^{ \pm} \mathbf{C K}\right]}
$$

and the corresponding pfaffian formula is ${ }^{6}$

$$
\mathbb{O}_{\ell}(z, \bar{z}, \alpha, \bar{\alpha})=\frac{1}{2} \sum_{ \pm} \frac{\operatorname{Pf}\left[\mathbf{C}^{-1}-\lambda^{ \pm} \mathbf{K}\right]}{\operatorname{Pf}\left[\mathbf{C}^{-1}\right]} .
$$

with

$$
\left[\mathbf{C}^{-1}\right]_{k l}=\sum_{n \geq 0}\left(\delta_{2 n+1+(-1)^{k} k+l}-\delta_{2 n+1+k+(-1)^{l} l}\right)
$$

\subsection{Integral representation for the matrix elements}

The operator expression (2.35) for the elements of the matrix $\mathbf{K}$ can be transformed into an integral by a Fourier transformation,

$$
\begin{aligned}
\mathcal{P}_{m n} & =\frac{1}{i g} \sum_{a=1}^{\infty} \frac{\sin a \phi}{\sin \phi} \int_{-\infty}^{\infty} d t \tilde{\Omega}_{\ell+n}^{-}(t) e^{-a t} \tilde{\Omega}_{\ell+m}^{+}(-t) \\
& =\frac{1}{i g} \int_{-\infty}^{\infty} d t \frac{\tilde{\Omega}_{\ell+n}^{-}(t) \tilde{\Omega}_{\ell+m}^{+}(-t)}{\cosh t-\cos \phi}
\end{aligned}
$$

where $\tilde{\Omega}_{j}^{ \pm}(t)$ is the Fourier image of $\Omega_{j}(u \pm i \epsilon)$,

$$
\begin{aligned}
\tilde{\Omega}_{k}^{ \pm}(\mp t) & =\int_{-\infty}^{\infty} \frac{d u}{2 \pi} e^{\mp i t u} \Omega_{k}(u \pm i 0) \\
& =g \int_{\mathbb{R}_{ \pm} i 0} \frac{d x(u)}{2 \pi} \frac{e^{\mp i g\left(x \tau_{ \pm}+\frac{1}{x} \tau_{\mp}\right)}}{x^{k+1}}, \quad \tau_{ \pm}=t \mp \xi=t \pm \log |z| .
\end{aligned}
$$

These integrals are computed by residues,

$$
\tilde{\Omega}_{k}^{ \pm}(\mp t)=\mp \alpha\left(\tau_{ \pm}\right) i g\left(i \sqrt{\frac{\tau_{-}}{\tau_{+}}}\right)^{\mp k} J_{k}\left(2 g \sqrt{\tau_{+} \tau_{-}}\right) .
$$

The Heaviside functions $\alpha\left(\tau_{ \pm}\right)$give a restriction of the lower bound in the integral in (2.39) to $t=|\xi|$. Hence the representation (1.31) of the matrix as an integral of a product of Bessel functions, which we write again for convenience,

$$
\mathrm{K}_{m n}=\frac{g}{2 i} \int_{|\xi|}^{\infty} d t \frac{\left(i \sqrt{\frac{t+\xi}{t-\xi}}\right)^{m-n}-\left(i \sqrt{\frac{t+\xi}{t-\xi}}\right)^{n-m}}{\cos \phi-\cosh t} J_{m+\ell}\left(2 g \sqrt{t^{2}-\xi^{2}}\right) J_{n+\ell}\left(2 g \sqrt{t^{2}-\xi^{2}}\right) .
$$

This completes the derivation of our main formula (1.29).

\footnotetext{
${ }^{6}$ If we approximate the Fredholm kernel with a degenerate one obtained by truncating the sum in (2.30) to $0 \leq m, n \leq N-1$, then $\operatorname{Pf}\left[\mathbf{C}^{-1}\right]=(-1)^{N}$.
} 


\section{The perturbative octagon}

Throughout this section we will assume that we are in the weak coupling regime. Within the perturbative expansion we are going to render the representation (1.29)-(1.31) more explicit and show that it matches the weak coupling results in $[2,18]$. For that we need to compute the perturbative expansion for the matrix elements $\mathrm{K}_{m n}$.

\subsection{Weak coupling expansion of the matrix $\mathrm{K}$}

In [2] it is claimed that the dependence of the octagon on the positions and on the polarisations of the four operators is only through the $j$-loop ladder integrals, which are evaluated as linear combinations of polylogarithms $[25]^{7}$

$$
F_{j}(z, \bar{z})=\sum_{s=0}^{j}\left(\begin{array}{c}
2 j-s \\
j
\end{array}\right) \frac{|\log z \bar{z}|^{s}}{s !} \frac{\operatorname{Li}_{2 j-s}(z)-\operatorname{Li}_{2 j-s}(\bar{z})}{z-\bar{z}}=\frac{1}{j !(j-1) !} f_{j}(z, \bar{z}) .
$$

In appendix A we obtain for the matrix elements (1.31) a double expansion in ladder integrals $f_{k}$ and the parameter $\xi=-\log \sqrt{z \bar{z}}$,

$$
\begin{aligned}
\mathrm{K}_{m+r, m}= & e^{-\xi} \sum_{p=0}^{\infty}\left(\begin{array}{c}
2 \ell+2 m+2 p+r \\
p
\end{array}\right) \frac{(-1)^{l+m}(i g)^{2 \ell+2 m+2 p+r+1}}{(\ell+m+p) !(\ell+m+p+r) !} \\
& \times \sum_{k=1}^{\left[\frac{r+1}{2}\right]}\left(\begin{array}{l}
r-k \\
k-1
\end{array}\right)|2 \xi|^{r-2 k+1} f_{\ell+m+p+k}(z, \bar{z}) \quad(r \geq 1) .
\end{aligned}
$$

The terms with nontrivial powers of $\xi$ are not consistent with the crossing invariance of the 4-point function. Fortunately it turns out that they do not show up in the traces $\operatorname{tr}\left[(\mathbf{C K})^{n}\right]$. The redundant $\xi$-dependence of the expansion (3.2) seems to be an artefact of the choice of the basis (2.30). That is, there is a rotation $O \mathbf{C K} O^{-1}$ which kills all the dependence on $\xi$ of the matrix elements, while preserving all traces. Assuming that this is the case, we replace the series for $\mathrm{K}_{n, m}$ with the truncated series obtained by removing all terms with non-trivial powers of $\xi .8$

Let us denote the truncated series by $\mathrm{K}_{n, m}^{\circ}$. For even values of $r$ all powers of $\xi$ in (3.2) are nontrivial and we get

$$
\mathrm{K}_{m+2 s, m}^{\circ}=0 .
$$

For odd powers of $r$ we denote $r=2 s-1$ with $s \geq 1$. Retaining from the sum in the second line of (3.2) only the term with $k=(r+1) / 2=s$ we get

$$
\mathrm{K}_{m+2 s-1, m}^{\circ}=e^{-\xi} \sum_{j=l+m+s}^{\infty}\left(\begin{array}{c}
2 j-1 \\
j-l-m-s
\end{array}\right) \frac{(-1)^{j-l-m}}{(j-s) !(j+s-1) !} g^{2 j} f_{j}(z, \bar{z}) .
$$

\footnotetext{
${ }^{7}$ In the notations of [25], $F_{j}(z, \bar{z})=-v \Phi^{(j)}(u, v)$.

${ }^{8}$ Note that setting $\xi=0$ in the ladder functions as well, simplifies the integral formula (1.31) and in particular imposes the opposite parity of the indices $m=n+1 \bmod 2$.
} 
Since $\operatorname{tr}\left[(\mathbf{C K})^{n}\right]=\operatorname{tr}\left[\left(\mathbf{C K}^{\circ}\right)^{n}\right]$, in order to obtain the perturbative series of the octagon up to $g^{4 N}$ it is sufficient to replace the semi-infinite matrix $\mathbf{K}^{\circ}$ to a $2 N \times 2 N$ matrix

$$
\mathbf{K}_{2 N \times 2 N}^{\circ}=\left(\mathrm{K}_{i j}^{\circ}\right)_{i, j=0,1, \ldots, 2 N-1} .
$$

The truncated determinant formula (2.36) reproduces the perturbative series for the octagon up to order $g^{4 N}$,

$$
\begin{aligned}
\mathbb{O}_{\ell} & =\frac{1}{2} \sum_{ \pm} \sqrt{\operatorname{Det}\left[1-\lambda^{ \pm} \mathbf{C}_{2 N \times 2 N} \mathbf{K}_{2 N \times 2 N}\right]}+o\left(g^{4 N+2 \ell}\right) \\
& =(-1)^{N} \frac{1}{2} \sum_{ \pm} \operatorname{Pf}\left[\mathbf{C}_{2 N \times 2 N}^{-1}-\lambda^{ \pm} \mathbf{K}_{2 N \times 2 N}^{\circ}\right]+o\left(g^{4 N+2 \ell}\right) .
\end{aligned}
$$

\subsection{The perturbative octagon as a determinant}

The formula (3.6) can be made aesthetically and conceptually more attractive if we repackage the $2 N \times 2 N$ matrices $\mathbf{C}_{2 N \times 2 N}$ and $\mathbf{K}_{2 N \times 2 N}$ in (3.6) as the $N \times N$ matrices

$$
\begin{array}{rlrl}
\mathcal{C}_{N \times N} & =\left(\mathcal{C}_{j k}\right)_{j, k=0, N-1}, & & \mathcal{C}_{j k} \equiv \mathrm{C}_{2 j, 2 k+1}=\delta_{j-k}-\delta_{j-k-1} \\
\mathcal{K}_{N \times N}=\left(\mathcal{K}_{i j}\right)_{i, j=0, \ldots, N-1}, & \mathcal{K}_{i j}=\mathrm{K}_{2 i+1,2 j}^{\circ} .
\end{array}
$$

Then due to (3.3) we have $\operatorname{tr}\left[\left(\mathbf{C K}^{\circ}\right)^{n}\right]=2 \operatorname{tr}\left[(\mathcal{C K})^{n}\right]$ and for any "cutoff" $N$ the following identity holds,

$$
(-1)^{N} \operatorname{Pf}\left[\left(\mathbf{C}^{-1}-\lambda \mathbf{K}^{\circ}\right)_{2 N \times 2 N}\right]=\operatorname{det}\left[(1-\lambda \mathcal{C K})_{N \times N}\right]
$$

The determinant formula for the perturbative octagon now takes the form ${ }^{9}$

$$
\mathbb{O}_{\ell}=\frac{1}{2} \sum_{ \pm} \operatorname{det}\left(1+\lambda^{ \pm} e^{-\xi} \mathcal{R}\right)_{N \times N}+o\left(g^{4 N+2 \ell}\right)
$$

where the matrix elements of $\mathcal{R}=-e^{\xi} \mathcal{C K}$ are given by a linear combination of ladders with rational coefficients,

$$
\begin{aligned}
\mathcal{R}_{i j}(z, \bar{z}, g) & =\sum_{p=\max (i+j+\ell, 1+j+\ell)}^{2 N} \mathcal{R}_{i j}(p) f_{p}(z, \bar{z}) g^{2 p}, \\
\mathcal{R}_{i j}(p) & =\frac{(-1)^{p-\ell}(2 p-1) !\left(2 p(2 i+\ell)\left(1-\delta_{i, 0}\right)-\delta_{i, 0}(p-j-\ell)(j+p)\right)}{(i-j+p) !(-i+j+p) !(-i-j-\ell+p) !(i+j+\ell+p) !} .
\end{aligned}
$$

\subsection{Expansion to nine loops}

In order to compare with the nine-loop result obtained in [2] by direct computation of the multiple integrals in the octagon series, we need to expand the determinant in (3.9) for

\footnotetext{
${ }^{9}$ For $\xi=0$ the determinant formula extends to any $g$ with $\mathbf{K}^{\circ}$ replaced by the (simplified) integral representation $(1.31)$.
} 


$$
\begin{aligned}
& \ell=0 \text { with } N=5 \text {, } \\
& \mathbb{O}_{\ell=0}=\frac{1}{2} \operatorname{det}\left(1+\lambda^{+} e^{-\xi} \mathcal{R}\right)_{5 \times 5}+\frac{1}{2} \operatorname{det}\left(1+\lambda^{-} e^{-\xi} \mathcal{R}\right)_{5 \times 5} \\
& =1+\mathcal{X}_{1}\left(f_{1} g^{2}-f_{2} g^{4}+\frac{1}{2} f_{3} g^{6}-\frac{5}{36} f_{4} g^{8}+\frac{7}{288} f_{5} g^{10}-\frac{7}{2400} f_{6} g^{12}+\frac{11}{43200} f_{7} g^{14}+\ldots\right) \\
& +\mathcal{X}_{2}\left(\frac{f_{1} f_{3}-f_{2}^{2}}{12} g^{8}-\frac{f_{1} f_{4}-f_{2} f_{3}}{24} g^{10}+\frac{7 f_{1} f_{5}-9 f_{3}^{2}+2 f_{2} f_{4}}{720} g^{12}+\frac{3 f_{3} f_{4}-2 f_{2} f_{5}-f_{1} f_{6}}{720} g^{14}+\ldots\right) \\
& +\mathcal{X}_{3}\left(\frac{f_{1} f_{5} f_{3}-f_{3}^{3}+2 f_{2} f_{4} f_{3}-f_{1} f_{4}^{2}-f_{2}^{2} f_{5}}{34560} g^{18}\right)+o\left(g^{20}\right),
\end{aligned}
$$

with

$$
\mathcal{X}_{n}=\frac{\lambda_{+}^{n}+\lambda_{-}^{n}}{2} e^{-n \xi},
$$

which matches completely the result obtained in [2].

\subsection{Fishnets}

In [18], the octagon was expanded in a basis of minors of the matrix (1.4). In particular, the lowest loop order $n$-particle contribution is proportional to the determinant of the matrix (1.4) restricted to the first $n$ rows and columns, which has been identified in [19] with the Feynman integral for an $n \times n$ fishnet,

$$
\mathbb{D}_{\ell=0}=\sum_{N=0}^{\infty} \mathcal{X}_{N} g^{2 N^{2}}\left(C_{N}+o\left(g^{2}\right)\right), \quad C_{N}=\frac{\operatorname{det}\left(\left[f_{i+j+1}\right]_{i, j=0, \ldots, N-1}\right)}{\prod_{i=0}^{N-1}(2 i) !(2 i+1) !} .
$$

This property of the octagon is obvious from our determinant representation (3.9), which can be written as a sum over minors of the matrix $\mathcal{R}$, eq. (1.35),

$$
\begin{aligned}
\mathbb{O}_{\ell=0} & =\sum_{N=0}^{\infty} \mathcal{X}_{N} \sum_{\substack{0 \leq i_{1}<\ldots<i_{N} \\
0 \leq j_{1}<\ldots<j_{N}}} \operatorname{det}\left(\left[\mathcal{R}_{i_{\alpha} j_{\beta}}\right]_{\alpha, \beta=1, \ldots, N}\right) \\
& =\sum_{N=0}^{\infty} \mathcal{X}_{N}\left(\operatorname{det} \mathcal{R}_{N \times N}+o\left(g^{2}\right)\right) .
\end{aligned}
$$

Indeed, to the lowest order the determinant of the matrix $\mathcal{R}_{N \times N}$ is given by the fishnet integral normalised as in (3.13),

$$
\operatorname{det} \mathcal{R}_{N \times N}=C_{N} g^{2 N^{2}}+o\left(g^{2 N^{2}+2}\right) .
$$

\subsection{The null-square limit}

Here we check that the logarithm of the octagon in the light cone limit $z \rightarrow 0,1 / \bar{z} \rightarrow 0$ takes the form (1.37) claimed in [18]. We reproduce this behavior from our determinant formula (3.9). The light cone limit corresponds to large imaginary values of the angle $\phi$. It is convenient to parametrise $z$ and $\bar{z}$ with the variable $y=i(\pi-\phi) \rightarrow \infty$,

$$
z=-e^{-\xi-y}, \quad \bar{z}=-e^{-\xi+y} .
$$


The light cone limit corresponds to $y \rightarrow+\infty$. Furthermore in the light cone limit $\lambda^{ \pm}=-e^{y}$, $\mathcal{X}_{n}=(-1)^{n} e^{n y} e^{-n \xi}=\bar{z}^{n}$, and the logarithm of the octagon is

$$
\log \mathbb{O}_{\ell=0}=\operatorname{tr} \log (1+\bar{z} \mathcal{R})=\bar{z} \operatorname{tr} \mathcal{R}-\frac{\bar{z}^{2}}{2} \operatorname{tr} \mathcal{R}^{2}+\frac{\bar{z}^{3}}{3} \operatorname{tr} \mathcal{R}^{3}-\ldots
$$

Take the matrix $\mathcal{R}$ for $\ell=0$,

$$
\mathcal{R}_{i j}=\sum_{p=\max (i+j, 1)}^{\infty}(-1)^{p}(2 p-1) ! \frac{4 i p-(p-j)(p+j) \delta_{i, 0}}{\prod_{\varepsilon= \pm}(p+\varepsilon(i-j)) !(p+\varepsilon(i+j)) !} f_{p} g^{2 p}
$$

and substitute the expansion of the ladders in the light cone limit [25]

$$
\begin{aligned}
-v f_{p}(z, \bar{z}) & =\sum_{m=0}^{p} \sum_{n=0}^{p}\left(\begin{array}{l}
p \\
m
\end{array}\right)\left(\begin{array}{l}
p \\
n
\end{array}\right)\left(1-2^{1-m-n}\right) \frac{1+(-1)^{m+n}}{p} \\
& \times(m+n) ! \zeta(m+n)(y+\xi)^{p-m}(y-\xi)^{p-n}
\end{aligned}
$$

where $-v=-(1-z)(1-\bar{z}) \rightarrow \bar{z}=-e^{y+\xi}$. E.g.

$$
f_{1}=\xi^{2}-y^{2}-\frac{\pi^{2}}{3}, \quad f_{2}=-\frac{1}{2}\left(y^{2}-\xi^{2}\right)^{2}+\frac{1}{3} \pi^{2}\left(\xi^{2}-3 y^{2}\right)-\frac{7 \pi^{4}}{30}
$$

etc. In general $\bar{z} f_{n}$ is a polynomial in $y$ and $\xi$ of degree $2 n$.

If we want to evaluate $\tilde{\Gamma}(g)$ to $N$ loops, we compute the expansion of the first $2 N$ terms of the series (3.17) with the matrix $\mathcal{R}$ truncated to $N \times N$,

$$
\log \mathbb{O}=\bar{z} \operatorname{tr} \mathcal{R}_{N \times N}-\frac{\bar{z}^{2}}{2} \operatorname{tr} \mathcal{R}_{N \times N}^{2}+\frac{\bar{z}^{3}}{3} \operatorname{tr} \mathcal{R}_{N \times N}^{3}-\ldots+\frac{\bar{z}^{2 N}}{2 N} \mathcal{R}_{N \times N}^{2 N}+o\left(g^{2 N}\right) .
$$

Taking $N=10$ we find, with the help of Mathematica, an expression which is of the form (1.37)

$$
\log \mathbb{O}=-4 y^{2} \tilde{\Gamma}(g)+g^{2}\left(y^{2}+\xi^{2}\right)+\text { const. },
$$

with $\tilde{\Gamma}$ given by (1.39).

\section{CFT representation}

An important property of the sum over virtual particles used to solder the two hexagons into an octagon, eq. (1.17), is that the scattering of each particle with the other particles is taken into account by a local weight factor. As a consequence, the two-particle interaction is symmetric and the grand canonical sum can be mapped onto a Coulomb gas of dipole charges. We will show that this Coulomb gas appears as the expansion of a certain expectation value in a theory of a twisted chiral boson defined on the Zhukovsky plane. The twisted boson changes sign every time it circles one of the two branch points at $u= \pm 2 g$. We will also show that the vertex operators of the bosonic field can be mapped to bilinears of real fermions. 


\subsection{The octagon in terms of a free chiral boson}

We introduce a chiral gaussian field $\varphi(u)$ defined on the Zhukovsky plane. The field is defined in terms of a mode expansion over a complete set of functions $\psi_{n}(u)=x(u)^{n}$ $(n \geq 1)$, where $x=x(u)$ is the Zhukovsky map $u \rightarrow x(u)$,

$$
\begin{aligned}
\varphi(u) & =\hat{q}+\hat{p} \ln x(u)-\sum_{n \neq 0} \frac{J_{n}}{n} x(u)^{-n}, \\
{\left[J_{n}, J_{m}\right] } & =n \delta_{m+n, 0} ; \quad[\hat{p}, \hat{q}]=1 .
\end{aligned}
$$

The Fock space is build on the vacuum states $\langle 0|$ and $|0\rangle$ with the properties

$$
\begin{array}{ll}
J_{n}|0\rangle=0, & (n>0) ; \quad \hat{p}|0\rangle=0, \\
\langle 0| J_{n}=0, & (n<0) ; \quad\langle 0| \hat{q}=0,
\end{array}
$$

so that the two-point function is

$$
\langle\varphi(u) \varphi(v)\rangle=\ln (x(u)-x(v)), \quad|x|>|y|>1 .
$$

Let us denote by $\varphi^{(\varepsilon)}(u), \varepsilon= \pm 1$, the value of the gaussian field on each of the two sheets of the Riemann surface with a cut $[-2 g, 2 g]$. The mode decomposition for $\varphi^{(-)}(u)$ is given by (4.1) with $x \rightarrow 1 / x$. The 2-point functions of the pair of fields $\varphi^{(\varepsilon)}(u)$ are given by

$$
\left\langle\varphi^{\left(\varepsilon_{1}\right)}(u) \varphi^{\left(\varepsilon_{2}\right)}(v)\right\rangle=\ln \left(x^{\varepsilon_{1}}(u)-x^{\varepsilon_{2}}(v)\right) .
$$

The monodromy around a branch point exchanges $\varphi^{(+)}(u)$ and $\varphi^{(-)}(u)$ and is diagonalised by

$$
\phi(u)=\frac{\varphi^{(+)}(u)-\varphi^{(-)}(u)}{\sqrt{2}}, \quad \tilde{\phi}(u)=\frac{\varphi^{(+)}(u)+\varphi^{(-)}(u)}{\sqrt{2}} .
$$

We will call the field $\phi$, antisymmetric with respect to the inversion $x \rightarrow 1 / x$, twisted and the field $\tilde{\phi}$ untwisted. The two-point function of the twisted field is while the two-point function of the untwisted field is

$$
\langle 0|\tilde{\phi}(u) \tilde{\phi}(v)| 0\rangle=\frac{1}{2} \ln (x-y)\left(\frac{1}{x}-\frac{1}{y}\right)\left(x-\frac{1}{y}\right)\left(\frac{1}{x}-y\right)=\ln \left(\frac{u-v}{g}\right) .
$$

The two-point function of the normal ordered exponents of the twisted field reproduces the antisymmetric kernel (2.8)

$$
: e^{\phi(u)}:: e^{\phi(v)}:=K(u, v): e^{\phi(u)+\phi(v)}:
$$

The factor (1.20) is reproduced by

$$
H_{a b}(u, v)=\left\langle 0\left|: e^{\phi(u+i a / 2)+\phi(u-i a / 2)}:: e^{\phi(v+i b / 2)+\phi(v-i b / 2)}:\right| 0\right\rangle .
$$

For any operator made out of the oscillators (4.2) we define another vacuum expectation value as

$$
\langle\mathcal{O}\rangle=\left\langle 0\left|\mathcal{O} e^{-i \sqrt{2} g \xi J_{-1}-\frac{\ell}{\sqrt{2}} q}\right| 0\right\rangle
$$


so that

$$
\langle\phi(u)\rangle=\phi_{c}(u)=i g \xi(x-1 / x)-\ell \ln x .
$$

Furthermore define the operator ${ }^{10}$

$$
\boldsymbol{\Phi}(u)=\log \frac{g}{\sqrt{u^{2}-4 g^{2}}}+\phi(u)
$$

so that expectation value (4.10) of the vertex operator is exactly the function $\Omega_{\ell}(u)$ defined in $(2.20)$,

$$
\left\langle: e^{\Phi(u)}:\right\rangle=\Omega_{\ell}(u)=e^{\Phi_{c}(u)}
$$

while the two-point function reproduces the scalar kernel (2.8),

$$
\left\langle: e^{\Phi(u)}:: e^{\Phi(v)}:\right\rangle=\Omega_{\ell}(u) \Omega_{\ell}(v) \frac{x(u)-x(v)}{x(u) x(v)-1}=\hat{K}(u, v) .
$$

The integration measure (1.18) is given by

$$
\boldsymbol{\mu}_{a}(u)=\frac{1}{i g} \hat{K}(u+i a / 2, u-i a / 2) .
$$

The series (1.26) is obtained by expanding the following expectation value,

$$
\mathbb{O}_{\ell}=\frac{1}{2} \sum_{ \pm}\left\langle\exp \left[\lambda^{ \pm} \int \frac{d u}{2 \pi i g}: e^{\mathbf{\Phi}(u-i 0)}: \frac{1}{2 \cos \phi-2 \cos \partial_{u}}: e^{\mathbf{\Phi}(u+i 0)}:\right]\right\rangle .
$$

\subsection{Real fermion}

The exponential field

$$
\Psi(u)=: e^{\phi(u)}:
$$

behaves as a real fermion. The correlation function of $2 n$ such fermions is the pfaffian of the two-point functions

$$
\left\langle\Psi\left(u_{1}\right) \ldots \Psi\left(u_{2 n}\right)\right\rangle=\operatorname{Pf}\left(\left[\frac{x\left(u_{j}\right)-x\left(u_{k}\right)}{x\left(u_{j}\right) x\left(u_{k}\right)-1}\right]_{i, j=1}^{2 n}\right) .
$$

The series (2.23) gives the free energy of these fermions. Thefree energy is the sum of all fermionic loops (the factor $1 / 2$ reflects the fact that the fermionic lines are non-oriented).

The analytic field $\boldsymbol{\Psi}(u)$ is completely determined by the mode expansion

$$
\boldsymbol{\Psi}(u)=\sum_{m \geq 0} \boldsymbol{\Psi}_{m} x(u)^{-m}, \quad\left\langle 0\left|\boldsymbol{\Psi}_{m} \boldsymbol{\Psi}_{n}\right| 0\right\rangle=\mathrm{C}_{m n} .
$$

\footnotetext{
${ }^{10}$ The shift of the gaussian field seems somewhat ad hoc. It can be obtained by defining the vertex operator without normal ordering and computing the singular part by point splitting by $\varepsilon$,

$$
\left\langle 0\left|e^{\phi(u)}\right| 0\right\rangle_{\epsilon}=e^{\frac{1}{2}\langle 0|\phi(u+\epsilon) \phi(u)| 0\rangle}=\left(\frac{\epsilon x^{\prime}(u)}{x^{2}-1}\right)^{1 / 2}=\frac{\sqrt{\epsilon} / \sqrt{g}}{x\left(1-1 / x^{2}\right)}=\sqrt{\frac{\epsilon}{g}} \frac{g}{\sqrt{u^{2}-4 g^{2}}} .
$$


where $C_{m n}$ is given by (1.30). Indeed, we have

$$
\langle 0|\Psi(u) \Psi(v)| 0\rangle=\frac{x-y}{x y-1}=\sum_{m, n \geq 0} x^{-m} C_{m n} y^{-n} .
$$

The quadratic form in the exponent becomes in the discrete basis

$$
\frac{1}{2 i g} \int \frac{d u}{2 \pi} \boldsymbol{\Psi}(u-i 0) e^{\Phi_{c}(u-i 0)} \frac{1}{\cos \phi-\cos \partial_{u}} e^{\Phi_{c}(u+i 0)} \boldsymbol{\Psi}(u+i 0)=-\frac{1}{2} \sum_{m, n=0}^{\infty} \boldsymbol{\Psi}_{m} \mathrm{~K}_{n m} \boldsymbol{\Psi}_{n}
$$

with $\mathrm{K}_{m n}$ given by (2.35). In terms of the discrete fermionic modes the operator expression of the octagon reproduces the pfaffian representation of the octagon (2.37),

$$
\mathbb{O}_{\ell}=\frac{1}{2} \sum_{ \pm}\left\langle 0\left|e^{-\frac{\lambda^{ \pm}}{2} \sum_{m, n \geq 0} \boldsymbol{\Psi}_{m} \mathrm{~K}_{n m} \boldsymbol{\Psi}_{n}}\right| 0\right\rangle=\frac{1}{2} \sum_{ \pm} \frac{\operatorname{Pf}\left[\mathbf{C}^{-1}-\lambda^{ \pm} \mathbf{K}\right]}{\operatorname{Pf}\left[\mathbf{C}^{-1}\right]} .
$$

If the expectation value is understood as an integral over real grassmanian variables, then the first term of the pfaffian comes from the matrix of the quadratic form which produces the correlator (4.19).

\section{Conclusions}

In this paper we give an explicit expression for the octagon form factor, which is the building block in the construction of the class of four-point functions of heavy half-BPS operators considered in [2]. As it was recently discovered in [13], the higher terms in the $1 / N_{c}$ expansion of these functions can be expressed in terms of the octagon as well.

Our main result is the determinant formula for the octagon form factor, eq. (2.36), for any value of the gauge coupling. In this way the computation of the octagon is reduced to that of a single integral, eq. (1.31). The structure of this integral reminds the one for the generating function for the ladder Feynman integrals [26]. ${ }^{11}$ At weak coupling the traces (2.34) computed with the integral in question are linear combinations of products of ladder integrals. ${ }^{12}$

Our weak-coupling determinant formula, eqs. (1.34)-(1.35), provides an analytic expression for the coefficients in the complete perturbative expansion of the octagon (1.2). The perturbative expansion of the octagon is expressed in terms of ladder integrals and at each order satisfies the maximal transcendentality requirement.

The expansion of the octagon in ladders can be arranged, as pointed out by Coronado [18], as a linear combination of minors of the matrix of ladders (1.4). The set of linearly independent minors chosen in [18] and called there Steinmann basis is made of minors having subsequent indices in the vertical direction. The expansion of the octagon in minors is a direct consequence of our determinant representation (3.9)-(1.35) when written in the form (3.14).

\footnotetext{
${ }^{11}$ We thank L. Dixon for bringing to our attention ref. [26].

${ }^{12}$ The effective truncation of the double series (3.2) to (3.4) when inserted in the traces (2.34) is still an empirical observation awaiting a rigorous proof.
} 
We hope that the determinant formula (2.36) could give for the first time analytic access to the correlation functions at finite $g$. It is likely that for the generic choice of the parameters one can truncate the matrix CK to an $N \times N$ matrix with exponentially small in $N$ accuracy. The CFT representation of section 5 in terms of free bosons of fermions is potentially useful in exploring the strong coupling regime, along the lines of [22]. The strong coupling expression should be given by the functional determinant of the quantum spectral curve in the sense discussed in [22].

\section{Acknowledgments}

The authors are obliged to Till Bargheer, Frank Coronado and Pedro Vieira for discussions and useful exchanges. This research is partially supported by the Bulgarian NSF grant DN 18/1 and by the bilateral grant STC/Bulgaria-France 01/6, PHC RILA $2018 \mathrm{~N}^{\circ} 38658 \mathrm{NG}$.

\section{A Perturbative evaluation of the matrix elements}

Here we derive formula (3.2). The summation over $a$ and the Fourier transformation in section 2.4 can be done in the reverse order leading to the same result as in (2.42)

$$
\begin{aligned}
\mathcal{P}_{m^{\prime}, m} & =i^{m-m^{\prime}-1} g \sum_{a}|z|^{1-a} \frac{z^{a}-\bar{z}^{a}}{z-\bar{z}} \int_{|\xi|}^{\infty} d t e^{-a t}\left(\frac{\tau_{-}}{\tau_{+}}\right)^{\frac{m-m^{\prime}}{2}} J_{m+\ell}\left(2 g \sqrt{\tau_{-} \tau_{+}}\right) J_{m^{\prime}+\ell}\left(2 g \sqrt{\tau_{-} \tau_{+}}\right) \\
& =\frac{g}{2 i} \int_{|\xi|}^{\infty} d t \frac{\left(i \sqrt{\frac{\tau_{-}}{\tau_{+}}}\right)^{m-m^{\prime}} J_{m+\ell}\left(2 g \sqrt{\tau_{-} \tau_{+}}\right) J_{m^{\prime} \ell}\left(2 g \sqrt{\tau_{-} \tau_{+}}\right)}{\cosh t-\cos \phi}
\end{aligned}
$$

We next use that the product of Bessel functions with the same argument can be expanded in a power series, (8.442) in [27]

$$
J_{m}(2 y) J_{m^{\prime}}(2 y)=\sum_{p=0}^{\infty}\left(\begin{array}{c}
2 p+m+m^{\prime} \\
p
\end{array}\right) \frac{(-1)^{p} y^{m+m^{\prime}+2 p}}{(m+p) !\left(m^{\prime}+p\right) !} .
$$

We insert this expansion for $y=g \sqrt{\tau_{+} \tau_{-}}$in the first line of (A.1), change variables $\tau_{+} \rightarrow t, \tau_{-} \rightarrow t+2|\xi|$ (assuming that $\xi=-\log \sqrt{z \bar{z}}>0$ ), expand $(t+|2 \xi|)^{\ell+m+p}$ and take the integral over $t,(3.351-3).[27]$

$$
\begin{aligned}
\int_{|\xi|}^{\infty} e^{-a t} \tau_{+}^{\ell+m^{\prime}+p} \tau_{-}^{\ell+m+p}= & \left(\ell+m^{\prime}+p\right) !(\ell+m+p) ! \times \\
& \sum_{s} \frac{|\log z \bar{z}|^{s}}{s !}\left(\begin{array}{c}
2 \ell+m+m^{\prime}+2 p-s \\
\ell+m^{\prime}+p
\end{array}\right) \frac{|z|^{a}}{a^{2 l+m+m^{\prime}+2 p-s+1}} .
\end{aligned}
$$


The summation over $a$ in (A.1) produces polylogarithms

$$
\begin{array}{r}
\mathcal{P}_{m^{\prime}, m}=|z| i^{m-m^{\prime}-1} \sum_{p=0}^{\infty}(-1)^{p}\left(\begin{array}{c}
2 p+2 \ell+m+m^{\prime} \\
p
\end{array}\right) g^{2 p+2 \ell+m+m^{\prime}+1} \times \\
\sum_{s=0}^{p+m^{\prime}+\ell}\left(\begin{array}{c}
2 p+2 \ell+m+m^{\prime}-s \\
p+m^{\prime}+\ell
\end{array}\right) \frac{|\log z \bar{z}|^{s}}{s !} \mathrm{L}_{2 p+2 \ell+m+m^{\prime}-s+1}
\end{array}
$$

with $\quad \mathrm{L}_{n}=\mathrm{L}_{n}(z, \bar{z})=\frac{\operatorname{Li}_{n}(z)-\operatorname{Li}_{n}(\bar{z})}{z-\bar{z}}$.

The integral in (2.24) for arbitrary $n$ after summation in $a_{i}, \varepsilon_{i}$ reads (summation over all repeated indices assumed)

$$
\operatorname{Tr}(\mathbf{C K})^{n}=\prod_{i=1}^{n} C_{k_{i} m_{i}}\left(\mathcal{P}_{m_{i-1}, k_{i}}-\mathcal{P}_{k_{i}, m_{i-1}}\right), \quad m_{0}=m_{n}
$$

For $n=1$ we get

$$
\begin{aligned}
-\frac{1}{2} \operatorname{Tr}(\mathbf{C K}) & =-\sum_{m \geq 0} \mathrm{~K}_{m+1, m}=-\sum_{m \geq 0}\left(\mathcal{P}_{m+1, m}-\mathcal{P}_{m, m+1}\right) \\
& =e^{-\xi} \sum_{p \geq 0} \sum_{j \geq p+\ell+1}(-1)^{p}\left(\begin{array}{c}
2 j-1 \\
p
\end{array}\right) g^{2 j} F_{j}=e^{-\xi} \sum_{j \geq \ell+1}(-1)^{j-\ell-1}\left(\begin{array}{c}
2 j-2 \\
j-\ell-1
\end{array}\right) g^{2 j} F_{j}
\end{aligned}
$$

where $F_{j}$ is defined in (3.1). The final expression in (A.6) reproduces $e^{-\xi} I_{1, \ell}$ in formula (C.1) of [2].

Next we work out starting from (A.1) the difference $K_{m, m+r}$ in (2.39), for any $r \geq 1$. For that one derives the identity

$$
\begin{aligned}
& \frac{1}{s !}\left(\left(\begin{array}{c}
2 a+r-s \\
a+r
\end{array}\right)+(-1)^{r-1}\left(\begin{array}{c}
2 a+r-s \\
a
\end{array}\right)\right) \\
& =(-1)^{r-1} \sum_{k=1}^{\left[\frac{r+1}{2}\right]}\left(\begin{array}{l}
r-k \\
k-1
\end{array}\right) \frac{(a+k-1) !(a+k) !}{(s-r+2 k-1) ! a !(a+r) !}\left(\begin{array}{c}
2 a+2 k-(s-r+2 k-1) \\
a+k-(s-r+2 k+1)
\end{array}\right)
\end{aligned}
$$

applied for $a=p+m+\ell$. Then changing the variable $s \rightarrow s+r-2 k+1$ the sum over $s$ in (A.4) reproduces the ladder integral combination (3.1) and we obtain (3.2).

Open Access. This article is distributed under the terms of the Creative Commons Attribution License (CC-BY 4.0), which permits any use, distribution and reproduction in any medium, provided the original author(s) and source are credited.

\section{References}

[1] I. Kostov, V.B. Petkova and D. Serban, Determinant formula for the octagon form factor in $\mathcal{N}=4$ SYM, Phys. Rev. Lett. 122 (2019) 1601 [arXiv:1903.05038] [INSPIRE].

[2] F. Coronado, Perturbative four-point functions in planar $\mathcal{N}=4 S Y M$ from hexagonalization, JHEP 01 (2019) 056 [arXiv: 1811.00467] [INSPIRE]. 
[3] J.A. Minahan and K. Zarembo, The Bethe ansatz for $N=4$ superYang-Mills, JHEP 03 (2003) 013 [hep-th/0212208] [INSPIRE].

[4] N. Beisert et al., Review of AdS/CFT Integrability: An Overview, Lett. Math. Phys. 99 (2012) 3 [arXiv: 1012.3982] [INSPIRE].

[5] N. Gromov, V. Kazakov, S. Leurent and D. Volin, Quantum Spectral Curve for Planar $\mathcal{N}=4$ Super-Yang-Mills Theory, Phys. Rev. Lett. 112 (2014) 011602 [arXiv:1305.1939] [InSPIRE].

[6] N. Gromov, V. Kazakov, S. Leurent and D. Volin, Quantum spectral curve for arbitrary state/operator in $A d S_{5} / C F T_{4}, J H E P 09$ (2015) 187 [arXiv:1405.4857] [INSPIRE].

[7] B. Basso, S. Komatsu and P. Vieira, Structure Constants and Integrable Bootstrap in Planar $N=4$ SYM Theory, arXiv: 1505.06745 [INSPIRE].

[8] T. Fleury and S. Komatsu, Hexagonalization of Correlation Functions, JHEP 01 (2017) 130 [arXiv: 1611.05577] [INSPIRE].

[9] B. Eden and A. Sfondrini, Tessellating cushions: four-point functions in $\mathcal{N}=4 S Y M$, JHEP 10 (2017) 098 [arXiv: 1611.05436] [InSPIRE].

[10] T. Fleury and S. Komatsu, Hexagonalization of Correlation Functions II: Two-Particle Contributions, JHEP 02 (2018) 177 [arXiv:1711.05327] [INSPIRE].

[11] T. Bargheer, J. Caetano, T. Fleury, S. Komatsu and P. Vieira, Handling Handles: Nonplanar Integrability in $\mathcal{N}=4$ Supersymmetric Yang-Mills Theory, Phys. Rev. Lett. 121 (2018) 231602 [arXiv: 1711.05326] [INSPIRE].

[12] T. Bargheer, J. Caetano, T. Fleury, S. Komatsu and P. Vieira, Handling handles. Part II. Stratification and data analysis, JHEP 11 (2018) 095 [arXiv: 1809.09145] [INSPIRE].

[13] T. Bargheer, F. Coronado and P. Vieira, Octagons I: Combinatorics and Non-Planar Resummations, JHEP 08 (2019) 162 [arXiv:1904.00965] [INSPIRE].

[14] B. Basso, F. Coronado, S. Komatsu, H.T. Lam, P. Vieira and D.-l. Zhong, Asymptotic Four Point Functions, JHEP 07 (2019) 082 [arXiv:1701.04462] [INSPIRE].

[15] L.V. Kantorovich and V.I. Krylov, Approximate methods of higher analysis (in Russian), Fizmatgiz Press (1962).

[16] G. Akemann and E. Kanzieper, Integrable Structure of Ginibre's Ensemble of Real Random Matrices and a Pfaffian Integration Theorem, J. Statist. Phys. 129 (2007) 1159 [math-ph/0703019] [INSPIRE].

[17] A. Borodin and E. Kanzieper, A note on the Pfaffian integration theorem, J. Phys. A 40 (2007) F849 [arXiv:0707.2784] [INSPIRE].

[18] F. Coronado, Bootstrapping the simplest correlator in planar $\mathcal{N}=4 S Y M$ at all loops, arXiv: 1811.03282 [INSPIRE].

[19] B. Basso and L.J. Dixon, Gluing Ladder Feynman Diagrams into Fishnets, Phys. Rev. Lett. 119 (2017) 071601 [arXiv:1705.03545] [INSPIRE].

[20] Ö. Gürdoğan and V. Kazakov, New Integrable 4D Quantum Field Theories from Strongly Deformed Planar $\mathcal{N}=4$ Supersymmetric Yang-Mills Theory, Phys. Rev. Lett. 117 (2016) 201602 [Addendum ibid. 117 (2016) 259903] [arXiv: 1512.06704] [INSPIRE].

[21] A.B. Zamolodchikov, 'Fishnet' diagrams as a completely integrable system, Phys. Lett. B 97 (1980) 63 [INSPIRE]. 
[22] Y. Jiang, S. Komatsu, I. Kostov and D. Serban, Clustering and the Three-Point Function, J. Phys. A 49 (2016) 454003 [arXiv: 1604.03575] [InSPIRE].

[23] D. Chicherin, A. Georgoudis, V. Gonçalves and R. Pereira, All five-loop planar four-point functions of half-BPS operators in $\mathcal{N}=4$ SYM, JHEP 11 (2018) 069 [arXiv:1809.00551] [INSPIRE].

[24] E.M. Rains, Correlation functions for symmetrized increasing subsequences, math. CO/0006097.

[25] N.I. Usyukina and A.I. Davydychev, Exact results for three and four point ladder diagrams with an arbitrary number of rungs, Phys. Lett. B 305 (1993) 136 [INSPIRE].

[26] D.J. Broadhurst and A.I. Davydychev, Exponential suppression with four legs and an infinity of loops, Nucl. Phys. Proc. Suppl. 205-206 (2010) 326 [arXiv:1007.0237] [InSPIRE].

[27] I.S. Gradshteyn and I.M. Ryzhik, Table of Integrals, Series, and Products, seventh edition, Academic Press (2007). 